\title{
Inductive or deductive? Research by maxillofacial surgeons
}

\author{
Soung Min Kim, BA, DDS, MSD, PhD ${ }^{1,2}$ \\ Section Editor of JKAOMS \\ ${ }^{1}$ Oral and Maxillofacial Microvascular Reconstruction LAB, Brong Ahafo Regional Hospital, Sunyani, Ghana, \\ ${ }^{2}$ Department of Oral and Maxillofacial Surgery, Dental Research Institute, School of Dentistry, Seoul National University, Seoul, Korea
}

Hundreds to thousands of scientific papers are published every day, most by renowned and competent reviewers as they evaluate and accredit each one according to their own standards. As oral and maxillofacial surgeons who perform clinical treatment and surgery every day, we have a scientific way of thinking and acting, and based on these skills, we publish experiences and records while evaluating and criticizing the experiments and results of others at the same time.

According to Röhrig et al. ${ }^{1}$, medical research is classified into primary and secondary research. Primary research includes basic, clinical, and epidemiological research, and secondary research includes meta-analysis and review (systematic and simple or narrative), such as cross-sectional, longitudinal, trend, cohort, and panel studies.

Basic research seeks to advance the frontiers of knowledge without a defined goal of utility or specific purpose and includes the development and improvement of analytical procedures. This pure research is also known as experimental research, whereas experiments in clinical research are also known as interventional and observational study ${ }^{1}$. Experimental or interventional studies involve an active attempt to change a disease determinant, such as an exposure or a behavior, or the progress of a disease through treatment. This is similar in design to experiments in other sciences. A clinical trial is a form of research to test new treatments and is divided into different stages. Non-interventional or obser-

\footnotetext{
Soung Min Kim

Department of Oral and Maxillofacial Surgery, Dental Research Institute, School of Dentistry, Seoul National University, 101 Daehak-ro, Jongno-gu, Seoul 03080, Korea

TEL: +82-2-2072-0213

E-mail:smin5@snu.ac.kr

ORCID: https://orcid.org/0000-0002-6916-0489

(c) This is an open-access article distributed under the terms of the Creative Commons Attribution Non-Commercial License (http://creativecommons.org/ licenses/by-nc/4.0/), which permits unrestricted non-commercial use, distribution, and reproduction in any medium, provided the original work is properly cited. Copyright (C) 2021 The Korean Association of Oral and Maxillofacial Surgeons. All rights reserved.
}

vational studies include comparative management, and the treatment is exclusively according to the doctor's discretion. Meanwhile, in epidemiological research or observational studies, researchers observe subjects and measure variables of interest. Assignment of subjects into treated and untreated groups is beyond the control of the researcher.

Before becoming clinicians, we must ask ourselves, "Are we deviating from a scientific and rational way of thinking?" As scientists, we need to evaluate data while coping with different circumstances. Therefore, in this issue, I would like to describe the two most basic approaches for methods of research: inductive and deductive. Although they can be complementary, these two approaches are quite different, and the relationship between theory and research differs for each approach.

The inductive method is cultural anthropology, deriving general facts from individual facts. The inductive method begins with research and establishing a theory, then investigates and observes to find a generalized theory. In the inductive method, the researcher begins by collecting relevant data for the research topic. Once a substantial amount of data has been collected, the researcher will develop an empirical generalization, stepping back to get an overview. In the early stages of inductive research, the researcher looks for preliminary patterns and regularities in the data, aiming to develop a tentative theory that could explain those patterns and regularities. Thus, the inductive approach starts with a set of observations and then moves from those experiences to broader generalizations about those experiences. In other words, they move from data to theory, or from the specific to the general.

The deductive method, on the other hand, derives specific facts from general facts in social science, pre-theory, postinvestigation, theoretical hypothesis investigation, observation and generalization. The deductive approach involves the same steps described earlier for inductive research but reverses the order. The researcher begins with a compelling theory 


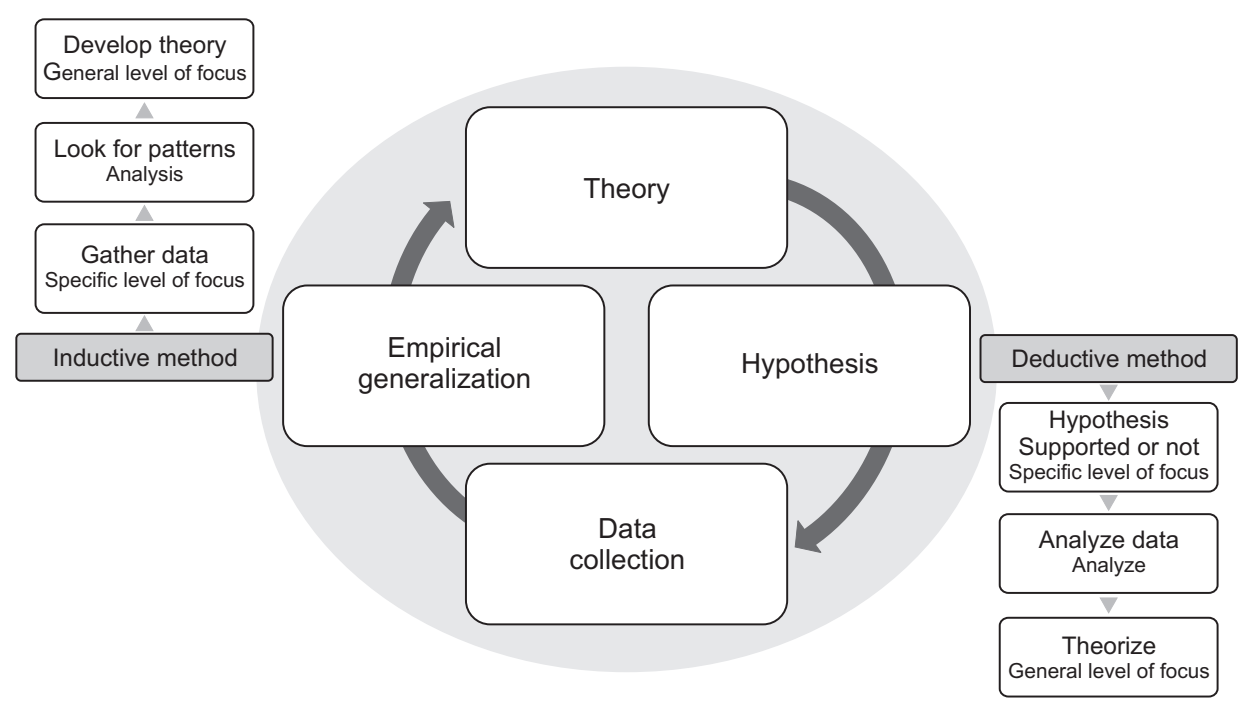

Fig. 1. Schematic chain of the approaches to inductive and deductive research.

Soung Min Kim: Inductive or deductive? Research by maxillofacial surgeons. J Korean Assoc Oral Maxillofac Surg 2021 and then tests its implications with data. That is, deductive research narrows information from a general to a more specific level. The deductive approach is typically associated with scientific investigation. The researcher studies what is known, analyzes the existing theories of the topic of interest, and then tests the hypotheses that emerge from the deductive methods.(Fig. 1)

We maxillofacial surgeons are both clinicians and scientists. Clinician-scientists are practicing professionals who engage in scientific research. By being involved in both fields, we have the unique opportunity to connect and exchange knowledge between research and practice, and as such, we are considered vital to the advancement of medical practice. By combining practice and research, we act as a bridge between distinct professional fields by transferring the most recent advancements from research to clinical practice and ensuring the clinical relevance of research, for example ${ }^{2}$. We must remind ourselves that medical knowledge is not acquired primarily for its own sake but for a specific purpose: the care of the sick ${ }^{3}$.

Understanding the cycles of deductive and inductive research methods can help an oral and maxillofacial surgeon take charge of an ideal clinical study through the integration of theoretical systems.

\section{Conflict of Interest}

No potential conflict of interest relevant to this article was reported.

\section{References}

1. Röhrig B, du Prel JB, Wachtlin D, Blettner M. Types of study in medical research: part 3 of a series on evaluation of scientific publications. Dtsch Arztebl Int 2009;106:262-8. https://doi.org/10.3238/ arztebl.2009.0262

2. de Groot E, Baggen Y, Moolenaar N, Stevens D, van Tartwijk J, Damoiseaux R, et al. Clinician-scientists in-and-between research and practice: how social identity shapes brokerage. Minerva 2021;59:123-37. https://doi.org/10.1007/s11024-020-09420-7

3. Swayne J. The problem with science-the context and process of care: an excerpt from remodelling medicine. Glob Adv Health Med 2012;1:78-87. https://doi.org/10.7453/gahmj.2012.1.1.014

How to cite this article: Kim SM. Inductive or deductive? Research by maxillofacial surgeons. J Korean Assoc Oral Maxillofac Surg 2021;47:151-152. https://doi.org/10.5125/jkaoms.2021.47.3.151 